\title{
Ultralightweight deformable mirrors
}

\author{
Keith Patterson* and Sergio Pellegrino \\ Graduate Aerospace Laboratories, California Institute of Technology, 1200 E California Blvd., \\ Pasadena, California 91125, USA \\ *Corresponding author: kdpatter@caltech.edu
}

Received 25 April 2013; revised 27 June 2013; accepted 1 July 2013; posted 1 July 2013 (Doc. ID 189333); published 22 July 2013

\begin{abstract}
This paper presents a concept for ultralightweight deformable mirrors, based on a thin substrate of optical surface quality, coated with continuous active layers that provide separate modes of actuation at different length scales. This concept eliminates any kind of stiff backing structure for the mirror surface and exploits microfabrication technologies to provide tight integration of the active materials into the mirror structure, to avoid actuator print-through effects. Proof-of-concept, $10 \mathrm{~cm}$ diameter mirrors with an areal density of $0.6 \mathrm{~kg} / \mathrm{m}^{2}$ have been designed, built, and tested to measure their shape-correction performance and verify the finite-element models used for design. The low-cost manufacturing scheme involves low-temperature processing steps (below $140^{\circ} \mathrm{C}$ ) to minimize residual stresses, does not require precision photolithography, and is therefore scalable to larger diameters depending on application requirements. (c) 2013 Optical Society of America

OCIS codes: (220.1000) Aberration compensation; (220.1080) Active or adaptive optics; (220.4610)

Optical fabrication; (230.2090) Electro-optical devices; (230.4040) Mirrors; (230.4170) Multilayers.

http://dx.doi.org/10.1364/AO.52.005327
\end{abstract}

\section{Introduction}

Recent advances in mirror technologies have started a transformation in the architecture of space-based telescopes. Compare, for example, the monolithic primary mirror of the Hubble Space Telescope, with a diameter of $2.4 \mathrm{~m}$ and an areal density of $\approx 183 \mathrm{~kg} / \mathrm{m}^{2}$ [1], to the segmented aperture of the James Webb Space Telescope, with an overall diameter of $6.6 \mathrm{~m}$ and consisting of 18 lightweight beryllium mirrors with an areal density of $\approx 20 \mathrm{~kg} / \mathrm{m}^{2}$, each mounted on a set of mechanical actuators that provide rigid-body pointing and a single curvature adjustment capability [2]. Two key advances that made this larger and much lighter aperture possible were the use of a folding architecture and the use of wavefront sensing and control of the mirror surface error.

Further advances in technology are expected to enable even larger telescopes [3] . A recent study of large space apertures, sponsored by the Keck

$1559-128 \mathrm{X} / 13 / 225327-15 \$ 15.00 / 0$

(C) 2013 Optical Society of America
Institute of Space Studies [4], put forward the concept of forming large mosaic mirrors through on-orbit self-assembly of identical active mirror segments mounted on modular, low-cost spacecraft (mirrorcraft). Building on recent developments in autonomous self-assembly in space [5-7], the mirrorcraft would dock and become mechanically connected to one another, and the mirrors' shapes would then be adjusted to form a single coherent surface. An on-orbit demonstration of identical, active lightweight mirrors mounted on CubeSats is currently under development [8]. The current state of the art in primary mirrors is the active hybrid mirror technology [9], with an areal density of $\approx 10 \mathrm{~kg} / \mathrm{m}^{2}$ and a wavefront correction capability of the order of $20 \mu \mathrm{m}$, which sets the standard for further developments.

The approach chosen in the present research, first presented in $[10,11]$, eliminates any kind of stiff backing structure for the mirror surface and exploits microfabrication technologies to provide tight integration of the active materials into the mirror structure, to avoid actuator print-through effects. As well as decreasing the mass of the mirror, the use of thinner structures results in a larger wavefront 
control authority than previous schemes, which can enable the modular telescope architectures envisaged above. Opening the design space to flexible, ultralightweight mirrors also has the effect of making them more prone to dynamic excitation, which then raises potential issues in relation to acoustic loading and shock during launch. These issues will be separately addressed in a forthcoming mission by means of new launch packaging solutions for the mirrors [8].

This paper presents the first step in such a scalable ultralightweight mirror technology that has been demonstrated at the $10 \mathrm{~cm}$ diameter scale. The proposed mirrors are based on an active laminate that contains several actuation layers able to provide separate modes of actuation at the different length scales.

The paper is organized as follows: Section 2 provides a review of the current state of the art of relevant mirror technologies. Section 3 describes various aspects of the proposed mirror concept. Section 4 describes the mirror shape control methodology. Section 5 presents a set of experiments carried out on a prototype $10 \mathrm{~cm}$ diameter mirror, and Section $\underline{6}$ concludes the paper.

\section{Background}

There are several approaches for deforming mirrors by means of actuators. These approaches can be broadly divided into three categories, depending on the geometric arrangement of the actuators with respect to the mirror surface: (i) normal, (ii) parallel, and (iii) along the boundary of the mirror. In case (i), known as surface-normal actuation, an array of stack or piston actuators push and pull on the mirror surface to produce local bumps and dips. In case (ii), known as surface-parallel actuation, actuators attached to a mirror facesheet bend the mirror. Case (iii), boundary actuation, applies forces and/or torques on the mirror rim to produce distortions of the mirror interior. We expect that the future implementation of lighter and highly active space telescope systems will restrict the adoption of surface-normal actuation to smaller mirrors, typically the secondary or tertiary mirror of a telescope, whereas surfaceparallel actuation will become established as the dominant lightweight solution for larger mirrors such as primary segments. Boundary controlled mirrors are limited in the range of interior deformation modes that can be activated by the actuators.

Microelectromechanical-systems (MEMS)-based mirrors are well developed and are already used extensively in adaptive optics for ground telescopes, where they are used for correcting atmospheric turbulence, laser beaming, and other applications. Both surface normal and surface parallel actuation schemes have been developed. Deformable mirrors with diameters up to a few centimeters, hundreds to thousands of actuator channels, and actuation strokes of the order of several micrometers based on MEMS technology are marketed by Boston Micromachine Corp. and OKO Technologies [12,13]; silicon membrane mirrors actuated by PZT (lead zirconium titanate) have been fabricated with silicon technology [14]. These types of devices are quite limited in the size of their clear apertures, and the required fabrication processes are not suitable for scaling these designs up to larger diameters.

Larger surface-normal-actuation mirrors produced by Xinetics consist of lead magnesium niobate (PMN) stacks attached to a mirror facesheet. The Xinetics standard deformable mirrors have a diameter up to $200 \mathrm{~mm}$, maximum deflection range of 3-8 $\mu \mathrm{m}$, and 941 actuators at a spacing of $7 \mathrm{~mm}$ [15]. The adaptive secondary mirrors on multiple mirror telescope, Magellan Baade Telescope, and large binocular telescope use hundreds of surface-normal actuators. [16]

Boundary actuation has been investigated in large thin, lightweight shell mirrors [17] and also in secondary telescope mirrors where radial cantilever beams attached to the rim of a glass mirror were deflected by means of PZT actuators that impose longrange deformation while minimizing local dimpling effects for low-order modes [18].

Surface-parallel-actuation schemes have been studied extensively. Examples include PZT actuators laminated to glass or silicon substrates [19], piezoelectric strips or sheets bonded to the back of a thin shell [20-22], and schemes involving surface-parallel transducers integrated into the mirror structure [23]. Off-the-shelf deformable mirrors marketed by Cilas include a range of flat bimorph mirrors based on a symmetric arrangement of two glass plates enclosing two patterned piezoceramic plates; these mirrors have diameters up to $100 \mathrm{~mm}$ and 188 actuators that can provide a maximum curvature of $0.07 \mathrm{~m}^{-1}$. Cilas also markets a range of unimorph mirrors, with diameters up to $115 \mathrm{~mm}$ and 63 actuators, which can provide a maximum defocusing correction of $20 \mu \mathrm{m}$ peak to valley. For the active hybrid mirror technology, mentioned in Section 1, the reflective surface is provided by a $10-100 \mu \mathrm{m}$ thick nanolaminate foil, bonded to a lightweight silicon carbide structure. Hundreds of electrostrictive actuators are embedded within the structure to make adjustments to the surface figure, in the form of surface parallel actuation. Diameters up to $1 \mathrm{~m}$ have been demonstrated [9].

Extremely lightweight concepts have also been proposed for making mirrors from laminated polymer films [24,25]. A review of processes aimed at minimizing surface roughness, thickness variation, and thermal expansion has been compiled by de Blonk et al. [26].

\section{Mirror Concept}

The objective of this study is to develop deformable mirror designs that are lightweight, scalable, inexpensive, and with a sufficiently large shape correction dynamic range to allow the same base design to be used in many or all parts of a segmented aspheric mirror and/or to compensate for thermally induced distortion and long-term material effects such as creep and aging. These requirements are to ensure 
economies of scale and simpler architectures in future large telescopes.

For lightness, the approach of choice is surfaceparallel actuation. Specifically, the proposed approach is to develop designs and fabrication processes for laminated shell mirrors consisting of a stiff, thin layer with an optically smooth surface, bonded to one or more layers of active material. The active layers are coated with electrodes patterned in a way that provides optimal control of the mirror figure. The most aggressive approach for lightness would be to make the whole mirror structure out of active materials. This alternative was pursued at the early stages of the present study, but was later abandoned due to the difficulty of fabricating piezopolymer thin shells by replication of an optical quality surface.

The high-stiffness substrate included in the present scheme has the advantage of providing higher bending stiffness that aids shape retention without complex mounting fixtures and provides an initial shape for the mirror that is close to the desired optical figure. However, a disadvantage of this approach is that it decreases the dynamic range for shape adjustment.

\section{A. Active Materials}

A list of active materials suitable for the actuation layer of a deformable mirror based on the present concept, in order of increasing maximum actuation strain, is presented in Table 1 . The field that is used to control the actuation strain-electric, magnetic or temperature-is listed in the second column of the table, and, since precise control can be achieved most easily for electric voltage, our choice is focused on piezoelectric (both ceramic and polymer) and electrostrictive materials, and also dielectric elastomers. Two parameters that are used for the selection of actuators $[27,28]$ are the maximum actuation strain, i.e., the strain achieved by raising the control field to its highest safe value, and the blocked stress, i.e., the stress required to hold the material at zero strain overall while raising the control field to its highest safe value. The values of these parameters are listed in columns three and four.

Electrostrictives and piezoceramics were not pursued in the present research, although they do provide large actuation stresses with good strain linearity, and low hysteresis in the case of some electrostrictives. Their brittleness and high-temperature processing made it more desirable to use piezopolymers instead. The issues related to brittleness have been alleviated in the currently available macrofiber-composite actuators based on piezoceramic fibers bonded with epoxy and prestressed in a polymer package [29]; however, these are discrete devices that would lead to potential print-through problems and would also be difficult to integrate into a mirror concept that requires a large number of independent actuators. Thin coatings of ceramic actuator material do have potential for certain mirror applications, and are currently being developed by others. [30]

Piezoelectric polymers are a reasonable compromise between performance and ease of processing; hence they were selected for the present study. Their primary drawbacks are relatively low blocked stress and maximum actuation strain, but these issues can be addressed by designing a laminate with a sufficiently flexible passive layer. More specifically, the active material used in the present research was the copolymer of vinylidene fluoride and trifluoroethylene, $\mathrm{P}(\mathrm{VDF}-\mathrm{TrFE})$. This material is a semicrystalline, electroactive, thermoplastic fluoropolymer, which can be made piezoelectric by poling at room temperature. This process involves placing a large electric field through the material thickness in order to align the electric dipole domains within the material. Note that P(VDF-TrFE) is similar to the homopolymer, PVDF, but does not require mechanical stretching to achieve a piezoelectric crystalline state [31].

An extensive study has been done by Sandia National Laboratories [31] on the performance of PVDF and its copolymers under conditions similar to low Earth orbit and including: temperature variation, UV exposure, atomic oxygen, and other effects. Subject to certain restrictions on the allowable temperature range to prevent loss of piezoelectricity, approximately $-80^{\circ} \mathrm{C}$ to $90^{\circ} \mathrm{C}$, and the need to shield against atomic oxygen, the study concluded that this polymer is suitable for space applications. It should also be noted that an instrument on the New Horizons mission to Pluto utilizes films of PVDF to count dust particle impacts on its journey toward

Table 1. Candidate Active Materials

\begin{tabular}{|c|c|c|c|c|c|}
\hline Type & $\begin{array}{l}\text { Control } \\
\text { Field }\end{array}$ & Examples & $\begin{array}{l}\text { Free Strain } \\
(\%)\end{array}$ & $\begin{array}{l}\text { Blocked Stress } \\
(\mathrm{MPa})\end{array}$ & Notes \\
\hline Piezopolymers & Voltage & PVDF, P(VDF-TrFE) & 0.1 & 5 & $\begin{array}{l}\text { Easily controlled; low-temperature } \\
\text { processing }\end{array}$ \\
\hline Electrostrictives & Voltage & $\begin{array}{l}\text { PMN, lead magnesium } \\
\text { niobate-lead titanate, lead } \\
\text { lanthanum zirconium titanate }\end{array}$ & 0.1 & 100 & Low hysteresis; requires bias voltage \\
\hline Magnetostrictives & Magnetic & Terfenol-D & 0.2 & 70 & Require large magnetic fields \\
\hline Piezoceramics & Voltage & $\mathrm{PZT}, \mathrm{BaTO}_{3}$ & 0.2 & 100 & $\begin{array}{l}\text { Easily controlled; high-temperature } \\
\text { processing }\end{array}$ \\
\hline Shape memory alloys & Temperature & Nitinol, CuAlNi & 5 & 200 & Large strains; requires heating \\
\hline
\end{tabular}




\begin{tabular}{lcl}
\hline Material & Type & \multicolumn{1}{c}{ Notes } \\
\hline Si (single crystal) & Ceramic & $\begin{array}{l}\text { Benefits from existing fabrication technologies; readily available; } \\
\text { limited diameters but increasing over time }\end{array}$ \\
$\mathrm{SiC}$ & Ceramic & $\begin{array}{l}\text { Very stiff; can be made to any size } \\
\text { Traditional mirror material; new flexible electronics } \\
\text { Glass (FS, BK7, borosilicate, Zerodur, etc.) }\end{array}$ \\
Ceramic & Pisplay glasses could be suitable \\
Carbon fiber composites & Polymer & $\begin{array}{l}\text { Low thermal expansion; scalable using tape dispensing techniques; } \\
\text { surface roughness is open concern }\end{array}$ \\
$\mathrm{Al}, \mathrm{Be}$ & Metal & Ductile and easy to machine; low thermal stability; beryllium is toxic. \\
\hline
\end{tabular}

the outer solar system by measuring currents in the film [32].

\section{B. Substrate Selection and Active Laminate Construction}

The substrate should have high extensional stiffness, and yet its bending stiffness should be low to maximize the range of curvature changes that can be achieved for any given active material. Hence the material used for the substrate should be stiff, manufacturable in small thicknesses, and with low surface roughness. Alternately, it should be a material that can be polished to an optical quality finish and that will retain this finish in a space environment. Ideally, it would also have low density to reduce the overall mass of the mirror, have high thermal conductivity to prevent thermal gradients from distorting the shape, and be durable enough for handling and processing purposes. The surface roughness desired for optical wavelength mirrors is of the order of a few nanometers or less. Table 2 provides a list of several potentially suitable materials.

Assuming that the actuation layer is much thinner than the substrate, the curvature change, $\kappa$, can be estimated with Stoney's formula [33],

$$
\kappa=\frac{6 \varepsilon_{a} M_{a} t_{a}}{t_{s}^{2} M_{s}}=\frac{6 \sigma_{a} t_{a}}{t_{s}^{2} M_{s}},
$$

where $\epsilon_{a}$ is the free strain and $t_{s}, t_{a}$ and $M_{s}, M_{a}$ are the thicknesses and the biaxial moduli of the substrate and actuator, respectively. For an isotropic material, the biaxial modulus is $E / 1-\nu$, where $E$ is Young's modulus and $\nu$ is Poisson's ratio. The (biaxial) blocked stress, $\sigma_{a}=\epsilon_{a} M_{a}$, may be used in place of the actuation strain, if preferred.

In order to increase $\kappa$ without changing the blocked stress, the substrate thickness should be reduced or a softer substrate be chosen. However, there are some practical limits. First, when it was attempted to construct whole mirrors out of layers of $\mathrm{P}(\mathrm{VDF}-\mathrm{TrFE})$, it was found that the mirror would wrinkle (buckle) very easily unless it was held under a state of pretension. Second, high-modulus substrates that are too thin tend to bend due to gravity effects and become distorted due to fabrication stresses. Last, packaging such low-stiffness mirrors for launch is more challenging.

All of these effects can be analyzed with standard techniques, but a specific effect related to fabrication should be mentioned. In cases where the active material is poled after deposition onto the substrate, a residual poling strain, $\epsilon_{p}$, will remain due to the permanent reorientation of the dipole domains. If the poling strain is too large, this residual strain can cause the substrate to buckle into a cylindrical mode. The minimum (critical) thickness at the onset of buckling of a circular plate of radius $R$ is given by $[34,35]$

$$
t_{\text {crit }} \approx\left(\frac{1.05 \varepsilon_{p} M_{a} t_{a} R^{2}}{M_{s}}\right)^{1 / 3}
$$

Note that there could be other sources of residual strain beyond poling, for example, coating processes and thermal cycling. All such effects can be treated in a similar manner.

Because of their ready availability, high thermal conductivity, and excellent surface quality (roughness of $1-5 \mathrm{~nm}$ ), the substrates utilized to build mirror prototypes during the present study consisted of thin (100-200 $\mu \mathrm{m}$ thick), $100 \mathrm{~mm}$ diameter singlecrystal (1-0-0 orientation) silicon wafers, polished on both sides. The piezopolymer layer was spincoated onto the wafer. An organic solvent was used to dissolve the $\mathrm{P}(\mathrm{VDF}-\mathrm{TrFE})$ copolymer in powder form and create a resin with reasonably high viscosity. If the viscosity is too high, it is difficult to spincoat evenly, whereas a low viscosity produces layers that are too thin. This resin was poured onto the wafer surface, and the wafer was then rotated on a vacuum chuck for a set time and spin rate to produce a nearly uniform coating. The sample was then baked on a hot plate to boil off the solvent and anneal the thermoplastic polymer. This process was repeated multiple times to build up a film layer of the required thickness. The reason for building each active layer out of several thin coats is to lessen the probability of forming pinhole defects, as well as to attain thick enough layers for actuation purposes.

Each piezoelectric layer was coated on either side with a conductive film formed by vacuum sputtering a metal such as aluminum, copper, or gold. As an alternative to sputtering, thermal evaporation was also investigated, but the latter process may cause the active layer to heat up, which could introduce damage. Sputtering is preferable because it is a room temperature process. To avoid oxidation, the 
best metal is gold, but an intermediate layer of titanium or chromium is required in order to promote adhesion to the adjacent layers. Much care was needed to develop a successful process and to avoid defects such as pinholes, cracking, and delamination. Patterning of the metal film was done by covering the film with a shadow mask during sputtering; this works for simply connected geometries, but photolithographic techniques could be used for more complicated patterns.

After completing the mirror stack, it was necessary to change the state of the active material from paraelectric into piezoelectric. The method that was implemented consisted in directly applying a high voltage potential across the top and bottom electrodes of the whole stack, although corona poling methods could be used as an alternative [31]. At field levels of $50-100 \mathrm{MV} / \mathrm{m}$, the polymer used for the present study undergoes a transformation as local electric dipoles within the material align themselves to the external field. After removing the high voltage, the polymer is poled and thus ready to be actuated with lower voltages (approximately $25 \mathrm{MV} / \mathrm{m}$ ). If there are pinhole or crack defects present in the laminate, the high voltage may cause arcing across the defect and hence permanent damage. Typically, the metallization around the defect becomes vaporized as a result of local heating at the arc location, and the momentary electrical short is then reopened. Actually, the laminate is still largely functional after this occurrence, albeit with a small defective spot.

Measurements taken during an example poling process on a laminate consisting of a single $20 \mu \mathrm{m}$ thick $\mathrm{P}(\mathrm{VDF}-\mathrm{TrFE})$ copolymer layer over a $200 \mu \mathrm{m}$ thick, $100 \mathrm{~mm}$ diameter wafer are shown in Fig. 1. The electrode covered only the central $80 \mathrm{~mm}$ diameter of the mirror, to prevent electrical arcing around the edge. A digital image correlation setup was used to measure the deflection of the center of the mirror relative to its edge as well as the average curvature across the mirror. In Fig. 1(a) note the transition from a horizontal slope around $0 \mathrm{~V}$ to a strongly positive slope at the end of the poling process. Also note the significant residual strain, $\epsilon_{p}$, and overall deflection due to poling; this residual strain is larger than the typical linear actuation strain and must be accounted for in the mirror design. Luckily, the residual strain is nearly uniform, and hence results in a nearly axially symmetric deformation (defocus) of the laminate. Because $\mathrm{P}(\mathrm{VDF}-\mathrm{TrFE})$ is a thermoplastic, one possible way of countering the poling stress is to anneal this layer. Figure 2 shows the behavior of the same laminate after it had been poled. Cycles of -500 to $+500 \mathrm{~V}$ were applied and resulted in a nearly linear behavior with some observable hysteresis. The measured cycles were fairly repeatable, although the relatively low resolution of the measurements prevents us from reaching quantitative conclusions about potential accuracy of this laminate used as part of a deformable mirror.

Estimates of the curvatures that can be achieved by using a uniformly actuated, single layer of $\mathrm{P}$ (VDF-TrFE) with a thickness of $20 \mu \mathrm{m}$ on substrates of arbitrary thickness have been obtained by using Eq. (1); the results are plotted in Fig. 3. The theoretical lower limit imposed by buckling into a cylindrical

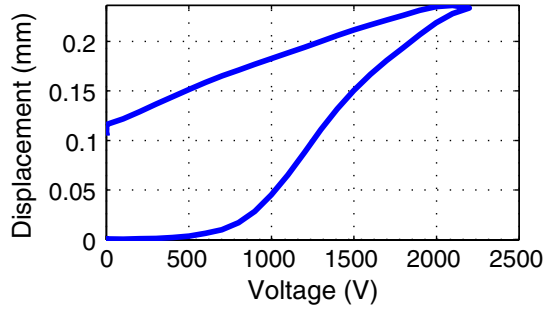

(a)

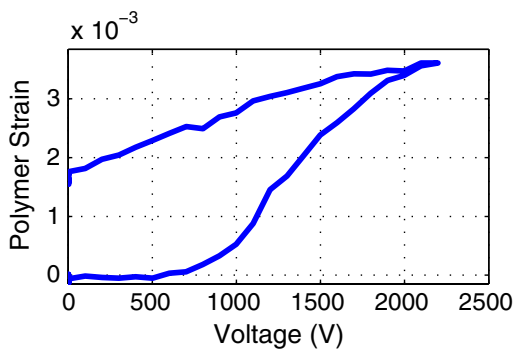

(b)

Fig. 1. Deformation of uniformly coated disk consisting of $20 \mu \mathrm{m} P(\mathrm{VDF}-\mathrm{TrFE})$ copolymer on a $200 \mu \mathrm{m}$ thick silicon wafer, during poling cycle. (a) Peak-to-valley deflection of mirror and (b) estimated piezoelectric strain in the polymer layer from Eq. (1), showing residual poling strain.

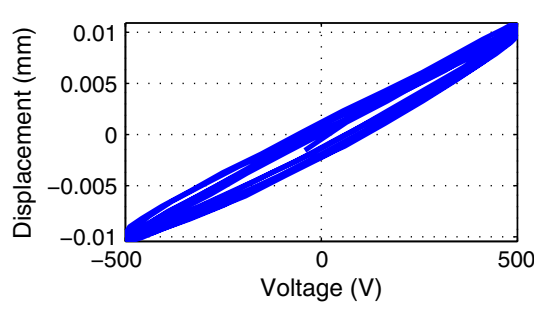

(a)

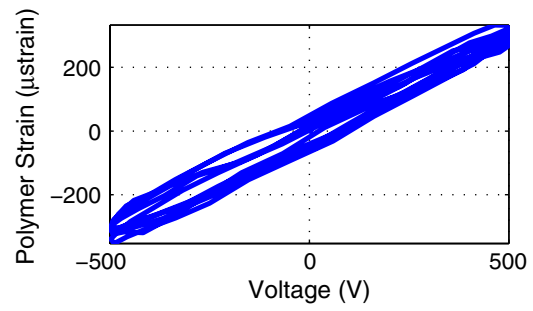

(b)

Fig. 2. Cyclical actuation of uniformly coated disk consisting of $20 \mu \mathrm{m} P(\mathrm{VDF}-\mathrm{TrFE})$ copolymer on $200 \mu \mathrm{m}$ thick silicon wafer, after poling process. (a) Peak-to-valley deflection and (b) estimated piezostrain in the polymer layer, from Eq. (1). 


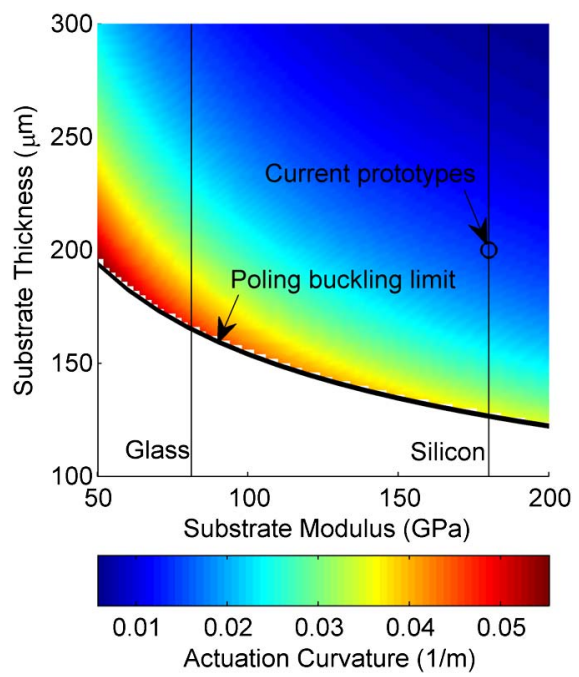

Fig. 3. Estimated actuation capability of $20 \mu \mathrm{m}$ thick P(VDFTrFE) coating as a function of substrate biaxial modulus and thickness. A practical lower limit is imposed by substrate buckling due to residual poling strain.

mode, predicted by Eq. (2), bounds the practical design space. Using this type of plot, an appropriate substrate material and thickness could be chosen based on a required actuation curvature and for a chosen active material.

Note that the poling fields have to be applied across the entire active layer, not only the patterned electrode regions, to prevent print-through of the electrode pattern into the mirror figure, due to poling stresses. In the present case this was achieved by depositing a thin, uniform coating of metal across the entire surface of the laminate, on top of the patterned electrodes, to act as a temporary poling electrode. A quick plasma etch after poling was then used to remove the uniform metal surface while leaving behind only the thicker, patterned metal underneath. If there are multiple active layers, the entire stack can be poled at once.

A summary of the layer thicknesses and processing steps for a complete mirror laminate is presented in Table $\underline{3}$, where layers $5-8$ can be repeated to provide greater shape control; see Subsection 3.C. Note that for curved mirrors the substrate requires additional processing (see Subsection 3.D), and also note that the reflective layer thickness can be increased to achieve thermal balance (see Subsection 3.E).

\section{Electrode Patterns}

To minimize print-through effects on the front (mirror) surface of the laminate, the active material layer is coated uniformly over the entire substrate, and then patterned electrodes are deposited on top. This provides a range of individually addressable actuator regions. See Fig. 4(a) for an example design with a glass substrate and annular electrode pattern. Complementary sets of actuation modes can be created by stacking multiple active layers within the laminate, each with its own set of unique electrode patterns. For example, the design in Fig. 4(b) shows large annular electrodes to provide broad, low-order corrections with relatively large stroke capability, while a high-density lattice of smaller electrodes in an upper active layer provides localized corrections at shorter length scales, albeit with lower stroke capability. See Fig. $\underline{5}$ for example influence functions generated by selected electrodes from the low- and high-density patterns of Fig. 4(b).

The topology and geometry of the high-density electrode pattern were selected from a trade study that considered several different patterns. Figure $\underline{6}$ shows six designs grouped into two families of patterns with varying actuator densities. The substrate is $200 \mu \mathrm{m}$ thick silicon, and the actuation layer is a continuous $20 \mu \mathrm{m}$ layer of $\mathrm{P}(\mathrm{VDF}-\mathrm{TrFE})$. The first pattern type, Figs. 6(a)-6(c), consists of rectangular strips arranged in a triangular lattice similar to that shown in Fig. 4. The second pattern type, Figs. 6(d)-6(f), is a hexagonal tessellation.

A finite-element model was set up for each of these patterns to compare their performance in making arbitrary corrections. The model was constructed in the software package Abaqus using S3T and S4T thermoelastic shell elements. Thermal expansion was used to simulate the piezoelectric effect, with the temperature field used as a substitute for the electric field, and the thermal expansion coefficient replacing the $d_{31}$ piezoelectric coefficient. The value of $d_{31}$ in the model was scaled so as to make a temperature change of $1 \mathrm{~K}$ equivalent to the application of $1 \mathrm{~V}$. This model was used to calculate the influence modes of each individual actuator (in units of $\mathrm{m} / \mathrm{V}$ ), by computing the mirror deflections due to a unit temperature change. The analysis assumed small deflections, and the material properties of the substrate and active layer were as given in Table $\underline{4}$. The results were

Table 3. Summary of Mirror Layers

\begin{tabular}{llcccl}
\hline Position & Mirror Layer & Material & Thickness $(\mu \mathrm{m})$ & Fabrication Order & Fabrication Method \\
\hline 1 & Reflective surface & $\mathrm{Al}$ or Au & 0.1 & 2 & Sputtering/evaporation \\
2 & Substrate & $\mathrm{Si}$ or Glass & 200 & 1 & COTS wafers \\
3 & Adhesion layer & $\mathrm{Ti}$ & 0.01 & 3 & Sputtering/evaporation \\
4 & Ground electrode & $\mathrm{Au}$ & 0.1 & 4 & Sputtering/evaporation \\
5 & Adhesion layer & $\mathrm{Ti}$ & 0.01 & 5 & Sputtering/evaporation \\
6 & Piezo layer & $\mathrm{P}(\mathrm{VDF}-\mathrm{TrFE})$ & 20 & 6 & Spin coating \\
7 & Adhesion layer & $\mathrm{Ti}$ & 0.01 & 7 & Sputtering/evaporation \\
8 & Electrodes & $\mathrm{Au}$ & 0.1 & 8 & Blanket sputtering + ion mill pattern etch back \\
\hline
\end{tabular}




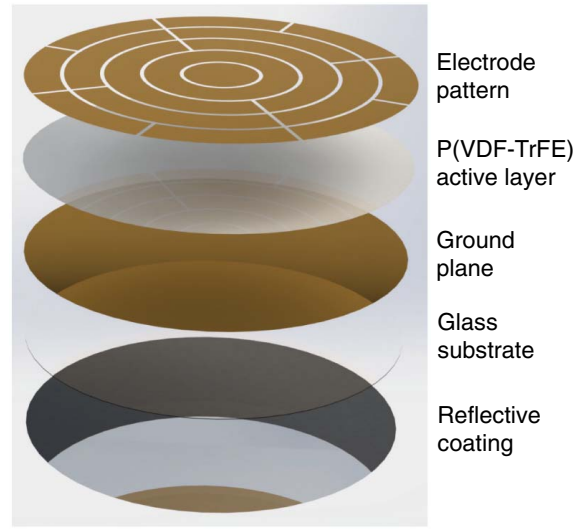

(a)

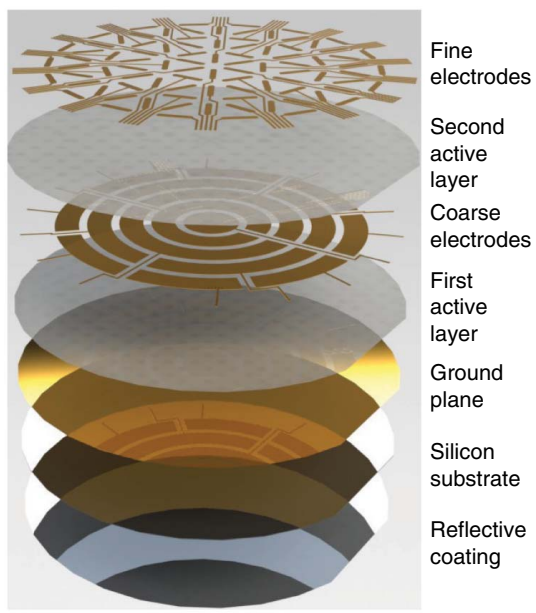

(b)

Fig. 4. Exploded views of example mirror layers: (a) single active layer and (b) double active layer.

then postprocessed in MATLAB to determine the performance of the mirror.

Shape errors in circular apertures are analyzed in terms of Zernike modes [36], i.e., a set of orthogonal polynomials defined over the unit disk. For each Zernike mode, all mirror designs were evaluated for how well they can correct an error in the shape of the chosen mode; the maximum RMS amplitude that can be corrected prior to saturating any of the actuators was also evaluated. The mathematical details are presented in Subsection 4.A, but the main results of this study are presented here.

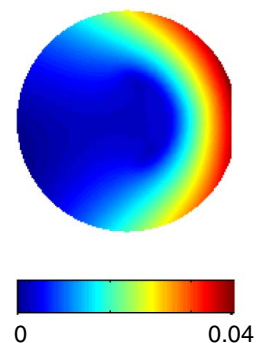

(a)

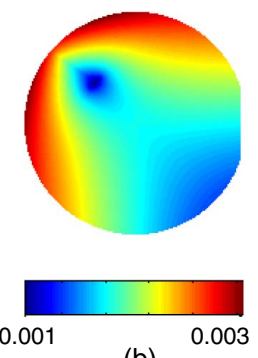

(b)
Fig. 5. Finite-element predictions of the influence of function shapes for selected electrodes from (a) coarse and (b) fine patterns of Fig. $\underline{4}$. Units are $\mu \mathrm{m} / \mathrm{V}$.

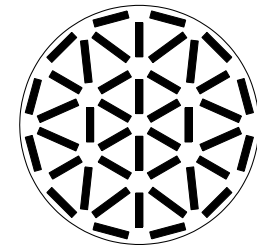

(a)

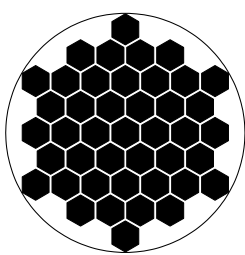

(d)

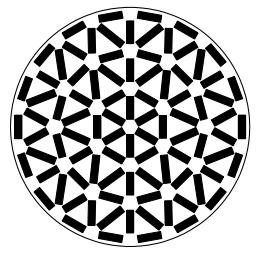

(b)

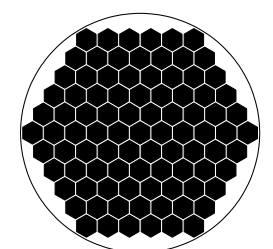

(e)

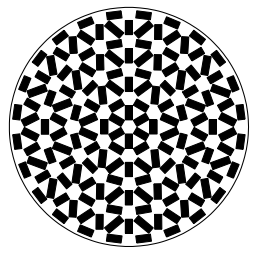

(c)

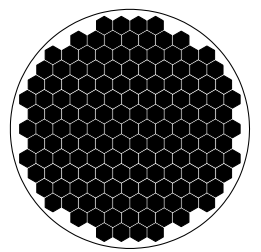

(f)
Fig. 6. Electrode design patterns: (a) - (c) triangular lattices with 42, 90, and 156 actuators; (d)-(f) hexagonal patch tessellations with 43,91 , and 151 actuators.

Table 4. Material Properties

\begin{tabular}{llc}
\hline Material & \multicolumn{1}{c}{ Property } & Value \\
\hline \multirow{3}{*}{$\mathrm{Si}$} & Biaxial modulus $\mathrm{M}$ & $180 \mathrm{GPa}$ \\
& Coefficient of thermal & $2.6 \mathrm{ppm} / \mathrm{K}$ \\
& expansion $\alpha$ & \\
& Elastic modulus $\mathrm{E}$ & $1.5 \mathrm{GPa}$ \\
$\mathrm{P}(\mathrm{VDF}-\mathrm{TRFE})$ & Poisson's ratio $\nu$ & 0.34 \\
& Biaxial modulus $\mathrm{M}$ & $2.3 \mathrm{GPa}$ \\
& Coefficient of thermal & $200 \mathrm{ppm} / \mathrm{K}(\mathrm{est})$. \\
& expansion $\alpha$ & \\
& Piezoelectric coefficient $d_{31}$ & $16 \mathrm{pm} / \mathrm{V}$ \\
$\mathrm{Al}$ & Biaxial modulus M & $120 \mathrm{GPa}$ \\
& Coefficient of thermal & $23 \mathrm{ppm} / \mathrm{K}$ \\
& expansion $\alpha$ & \\
\hline
\end{tabular}

Figure 7 shows the correctability for each of the first 30 Zernike modes, for the 6 high-density electrode patterns presented in Fig. 6 . Here, correctability is defined as the ratio of input RMS error to the output (corrected) residual RMS error, without considering the effects of actuator saturation.

For each Zernike mode, as the amplitude of the error increases, more and more of the actuation channels reach saturation at $500 \mathrm{~V}$. Hence, the relationship between the amplitude of the input RMS error in the chosen mode and the output (corrected) residual RMS error, shown schematically in Fig. $\underline{8}$, is nonlinear as more and more actuators become saturated. This becomes linear again with a slope equal to 1 when all actuators are saturated. The actual predictions for three important low-order modes (defocus, astigmatism, and coma) are shown in Fig. 9 . Note that after partial saturation the Hex 43 residual RMS is lower for coma than the Hex91, owing to the orientation of the mode with respect to the pattern of the electrodes.

The general trend is that, for any chosen pattern, increasing the actuator density can improve both the shape correction accuracy and available stroke, but this comes at the cost of increasing the complexity 


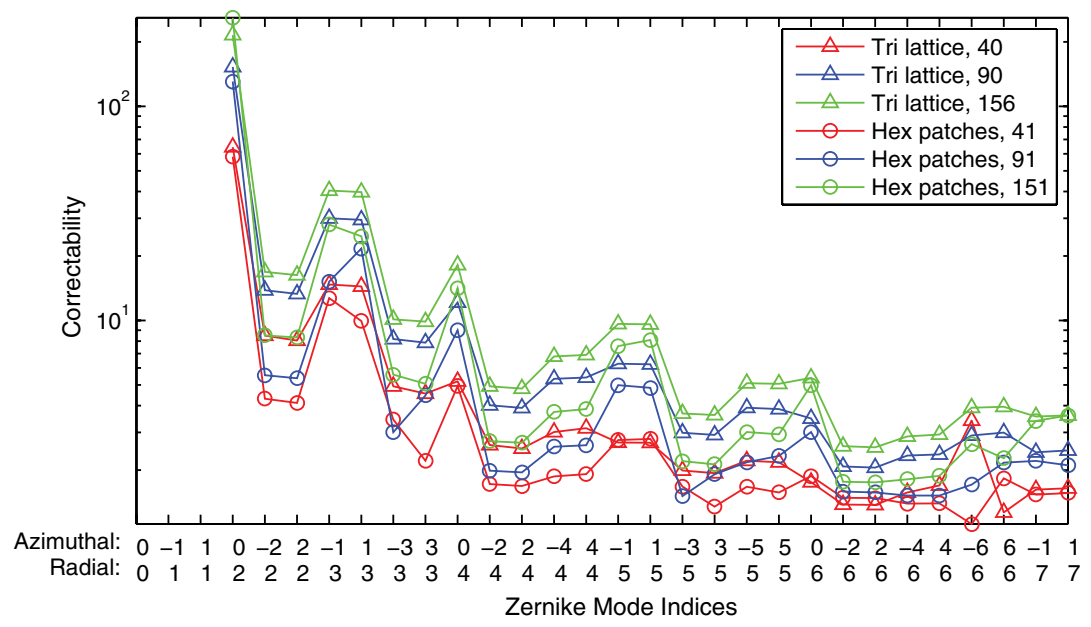

Fig. 7. Correctabilities of six actuation patterns in Fig. $\underline{6}$, for first 30 Zernike modes.

of the driving electronics. The choice of the electrode pattern is also important. Figure $\underline{6}$ shows that the triangular lattice pattern significantly outperforms the hexagonal pattern in terms of modal correctability, which can be explained by noting that placing the actuating strips in different orientations provides better control on the bending of the substrate in arbitrary directions. This aids in correcting shapes that require nonaxisymmetric deformations. Figure 9 shows that the hexagonal pattern is able to achieve larger strokes before saturation, which can be explained by noting that the hexagonal pattern provides a higher coverage of the surface and hence the total available actuation moment is higher. It would appear that a combination of these two patterns, where the unutilized area in the lattice patterns is filled in with triangular and hexagonal patches, may provide a good compromise between stroke and correctability. Clearly there is scope for future optimization of the electrode pattern.

\section{Curved Mirrors}

Glass or silicon wafers are excellent substrates for the mirrors; however they are usually manufactured to be nominally flat. In order to use deformable

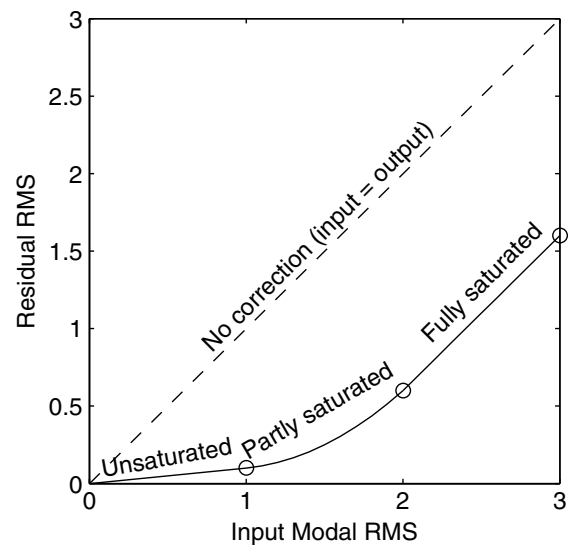

Fig. 8. General relationship between residual and input error in any chosen mode. mirrors in a primary aperture, it is desirable to introduce a base curvature to decrease the demand on the actuators. In the case of a low-cost telescope with a segmented primary mirror, identical spherical active segments with curvature equal to the average overall curvature of the aperture would be required [10].

One way to introduce a permanent curvature into a nominally flat substrate is by applying a stressed coating to the substrate, e.g., by depositing coatings while either cooling or heating the substrate. This works well up to a limit defined by Eq. (2), after
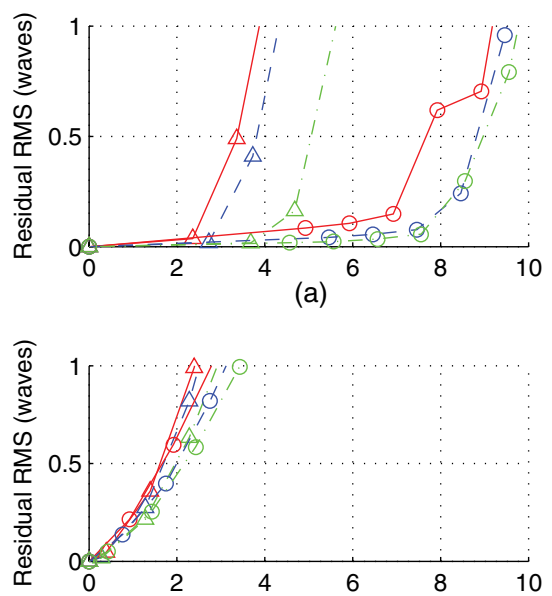

(b)

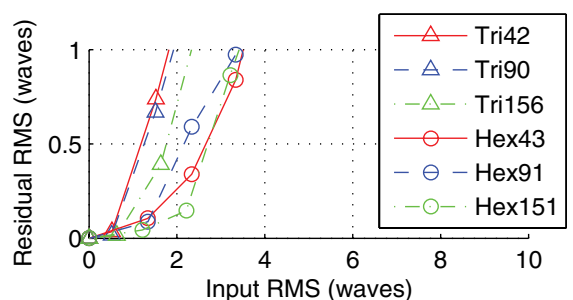

(c)

Fig. 9. Variation of residual RMS error with RMS error in three chosen modes for the six actuation patterns in Fig. 6. (a) Mode $Z_{2}^{0}$ defocus, (b) mode $Z_{2}^{-2}$ astimagtism, and (c) mode $Z_{3}^{-1}$ coma. The reference wavelength is $633 \mathrm{~nm}$. 
which the substrate will buckle from a spherical into a cylindrical shape [34]. To go beyond this limit, a boundary constraint could be used to hold the axisymmetric shape at higher levels of stress; however this comes at the cost of a reduced actuation range.

An alternative approach, suitable for glass substrates, is to support the substrate on a mold and raise the temperature to the glass transition temperature of the glass. This process, known as slumping, will cause the substrate to take the curvature of the mold (without sacrificing the optically smooth surface finish of the original wafer). It was used for the mirrors of the NuSTAR x-ray telescope [37]. It has been demonstrated that under carefully controlled conditions silicon can be slumped as well, at around $900^{\circ} \mathrm{C}[\underline{38}]$.

\section{E. Reflective Coating}

Polished silicon is reflective in the visible spectrum and its reflectivity is adequate for laboratory testing, but mirrors with high-throughput requirements need to be vacuum coated with a thin aluminum or silver film for visible light, or gold for infrared. A layer of a transparent ceramic oxide can be coated over the reflective film in order to protect these soft metals from damage and to prevent oxidation in the case of aluminum or silver. Depending on the coating thicknesses, care must be taken that residual coating stresses do not affect the thin substrate's figure, or do so in a desired, predetermined manner.

\section{F. Thermal Balancing}

A laminate made of layers of different materials will, in general, bend when it is subjected to bulk temperature changes. These effects can be analyzed and counteracted as discussed in this section.

Consider a laminate consisting of a substrate and any number of layers. Let $\alpha_{s}$ and $\alpha_{i}$ be the coefficients of thermal expansion of the substrate and the additional layers, respectively. It is assumed that all of the layers are much thinner than the substrate, and hence a simple estimate for the bending curvature resulting from a temperature change, $\Delta T$, can be constructed by substituting into Eq. (1) the thermal strain (relative to the substrate thermal strain), $\epsilon_{i}-\epsilon_{s}=\left(\alpha_{i}-\alpha_{s}\right) \Delta T$. Allowing for layers attached to both the top and the bottom of the substrate, the overall curvature can be obtained by superimposing their individual effects:

$$
\begin{aligned}
\kappa & =\sum_{i} \frac{6 s_{i}\left(\varepsilon_{i}-\varepsilon_{s}\right) M_{i} t_{i}}{t_{s}^{2} M_{s}}=\sum_{i} \frac{6 s_{i}\left(\alpha_{i}-\alpha_{s}\right) \Delta T M_{i} t_{i}}{t_{s}^{2} M_{s}} \\
& =\frac{6 \Delta T}{t_{s}^{2} M_{s}} \sum_{i} s_{i}\left(\alpha_{i}-\alpha_{s}\right) M_{i} t_{i},
\end{aligned}
$$

where $s_{i}=+1$ for a layer on top of the substrate and $s_{i}=-1$ for a layer on the bottom.

Thermal bending can be prevented by means of additional coating layers that balance the laminate thermal stresses. To do this, set the curvature to zero and add additional layers (either on top or bottom) with appropriate thicknesses until the overall thermal curvature is zero:

$$
0=\sum_{i} s_{i}\left(\alpha_{i}-\alpha_{s}\right) M_{i} t_{i}
$$

For example, a $100 \mu \mathrm{m}$ thick silicon substrate coated with a $20 \mu \mathrm{m}$ thick layer of PVDF can be balanced by means of a $3 \mu \mathrm{m}$ thick aluminum coating layer on the front, and buckling can be avoided by controlling the substrate temperature during deposition, to minimize the coating stress. The additional coating thickness required is small because of the higher stiffness of the metal coating relative to the polymer, and hence there is only a small increase in the bending stiffness of the mirror. Therefore the corresponding reduction in actuation capability is small. Since a metallic coating is required anyway for reflectivity, then the thermal balance issue can simply be addressed by ensuring that the reflective coating has the thickness required to balance out the actuation layers.

\section{G. Kinematic Mounting}

The surface parallel actuation scheme adopted in the present study is well suited for a kinematic mounting scheme, which mechanically constrains the six degrees of freedom (three translations, three rotations) of the mirror without adding any additional constraints that may put the mirror in an unintended state of self-stress. This approach allows the flexible optic to deform itself into any desired shape and to hold its shape without being affected by the behavior of the mount. However, great care is needed in designing a kinematic mount for an ultrathin plate or shell. For example, if mechanical flexures were employed to hold the mirror, any deformation introduced into the mirror by the flexures should have an amplitude of the order of optical wavelengths. Hence, the flexures would need to be designed to be so soft as to be impractical.

A simple and effective scheme to hold the mirror has been devised that takes advantage of the large through-thickness stiffness of the mirror. Note that the mirror can be pinched without significant deflections by applying equal and opposite forces on the front and back surfaces. The pinching forces need to be as close as possible to collinear, as any misalignment introduces shear and bending loads, which would induce significant deflections. Coalignment could be achieved with machined parts that have tight tolerances, but a simpler approach is to use self-aligning magnetic forces.

Figure 10 shows an implementation of this scheme. The mirror is attached to a printed circuit board (PCB) at three points, and at each of these points two polished, magnetized steel spheres pinch the mirror and hold it in place. The top sphere is unconstrained, and hence the magnetic field automatically aligns the upper and lower contact points. 


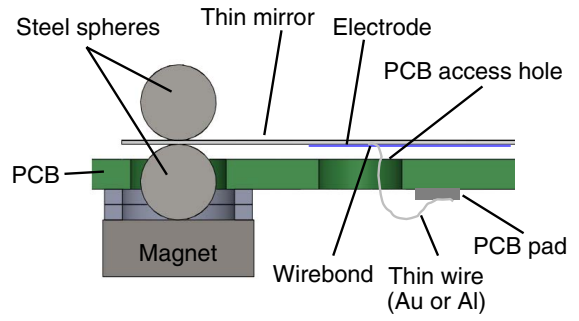

Fig. 10. Cross section of magnetic mounting scheme. Pairs of polished, magnetized spheres pinch the mirror at three places around the rim to hold it on a PCB. Tiny wire-bonded electrical connections jump from the board to the mirror.

Additional constraints would need to be added to restrain the remaining three in-plane degrees of freedom (two translations, one rotation), but, for the purpose of laboratory testing, friction at the pinched points is sufficient to hold the mirror in place.

Once the mirror is mounted on the PCB in this way, electrical connections are then made between the PCB and the mirror using very thin, prebuckled wires made of soft, ductile metal such as gold or aluminum, bonded to the back of the mirror. The properties of these wires are such that any forces or moments applied to the mirror laminate are sufficiently small to leave the mirror figure unaffected. Resistive heating due to the narrow wire cross section is not a concern because the wires carry only very short duration currents of microampere to milliampere levels. Figure 10 shows a schematic diagram of this connection scheme.

\section{Shape Control}

The RMS surface error (deviation from nominal shape) is a simple scalar measure of the shaperelated performance of a mirror that is particularly convenient for comparison purposes; it will be utilized as a criterion for mirror design [39]. It should be noted that the high-spatial-frequency components of the RMS error will be governed by the mirror surface roughness, which is related to manufacturing techniques and processes that cannot be addressed with shape correction. On the other hand, minimization of the low- to mid-frequency components of the RMS error may be achieved through the use of a sufficient number of actuators that bend the mirror into the desired shape. These effects are addressed in this section, together with the numerical techniques for mirror surface control.

\section{A. Influence Functions}

Consider $m$ sampling points (nodes) distributed on the surface of a general mirror surface, and an associated control system with $n$ actuators. Associated with the $i$ th actuator is a column vector, $\mathbf{a}_{i} \in \Re^{m}$, $i=1 \ldots n$, obtained from the nodal deflections of the mirror due to a unit input (e.g., $1 \mathrm{~V}$ ) to the $i$ th actuator, while all other actuators are turned off. This column vector is known as the influence vector of actuator $i$, since it determines the influence that the actuator has on the mirror surface. It is linearly independent from the other $n-1$ vectors, corresponding to the other actuators. Example influence functions are shown in Fig. $\underline{5}$. The influence vectors are assembled into the influence matrix, $\mathbf{A}$ :

$$
\mathbf{A}=\left[\begin{array}{llll}
\mathbf{a}_{1} & \mathbf{a}_{2} & \ldots & \mathbf{a}_{n}
\end{array}\right] \in \Re^{m \times n} .
$$

It is assumed that all deviations from the initial surface shape are small with respect to the diameter of the mirror. This assumption allows linear combinations of the influence vectors to be used to predict the mirror deflections. Hence, the influence matrix can be used to transform a control vector, $\mathbf{u} \in \Re^{n}$, consisting of the actuator input values, into a shape deflection vector, $\delta \in \Re^{m}$, which contains the deflection of all nodal points of the mirror. Thus, the control vector and shape deflection vector are related via the influence matrix by

$$
\mathbf{A u}=\delta .
$$

The correction of the mirror from its current shape, $\mathbf{s}_{1} \in \Re^{m}$, to a desired shape, $\mathbf{s}_{2} \in \Re^{m}$, requires a deflection $\delta=\mathbf{s}_{2}-\mathbf{s}_{1}$. This deflection vector will, in general, not belong to the range space of $\mathbf{A}$. Therefore, the appropriate control vector is obtained from the least squares solution of Eq. (6).

For generality, the nodal deflections are weighted by appropriate surface areas, $S_{i}$, to make the shape control formulation independent of meshing or sampling nonuniformities. In the present study, the values to $S_{i}$ were found by calculating the Voronoi area [40] surrounding each node. These area weights are arranged along the diagonal of a matrix, $\mathbf{W} \in \Re^{m \times m}$, and Eq. ( $\underline{6})$ is then modified to

$$
\mathbf{W A u}=\mathbf{W} \delta .
$$

The weighted, least-squares solution of Eq. (7) can be calculated by using the QR factorization or other methods, and software packages such as MATLAB have built in functionality to compute these solutions efficiently. If the available actuator inputs are constrained to a certain range, then a constrained, weighted, linear least squares solution would be required in order to find the optimal $\mathbf{u}$.

Once the solution $\mathbf{u}$ has been determined, the difference between the approximation and the original is the residual vector or residual shape error, $\mathbf{r}=\mathbf{A u}-\delta \in \Re^{m}$, or, accounting for the weights in the residual,

$$
\hat{\mathbf{r}}=\mathbf{W A u}-\mathbf{W} \delta \in \Re^{m} .
$$

For convenience, the weights in $\mathbf{W}$ can be redefined as the square roots of $S_{i}$ nondimensionalized by the total mirror surface area. Thus, the 2-norm of $\hat{\mathbf{r}}$ (usually with piston, tip, and tilt removed) is then equivalent to the RMS surface error: 


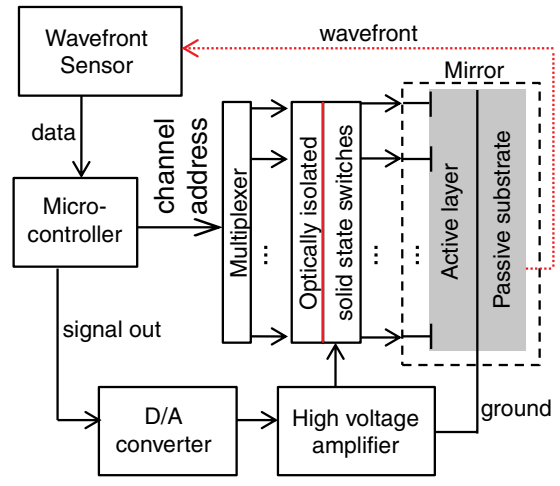

(a)

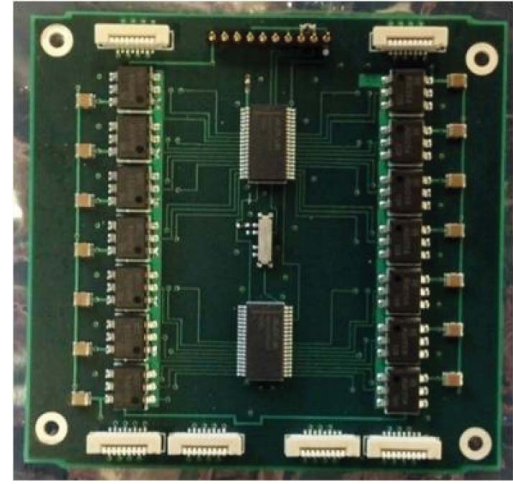

(b)

Fig. 11. (a) Schematic diagram of control system showing wavefront feedback from the mirror passed to a controller; the controller then uses a high-voltage amplifier and multiplexer to apply a new set of voltages to the mirror electrodes. (b) Photograph of a $10 \mathrm{~cm} \times 10 \mathrm{~cm}$ prototype high-voltage multiplexer board populated with solid-state switches is shown on the right; it is capable of running 42 channels to $\pm 500 \mathrm{~V}$.

$$
\begin{aligned}
\|\hat{\mathbf{r}}\|_{2}=\sqrt{\hat{\mathbf{r}}^{T} \hat{\mathbf{r}}} & =\sqrt{(\mathbf{A u}-\delta)^{T} \mathbf{W}^{2}(\mathbf{A u}-\delta)} \\
& \equiv \sqrt{\frac{\sum_{i} r_{i}^{2} S_{i}}{\sum_{i} S_{i}}}
\end{aligned}
$$

\section{B. Control Implementation}

To be consistent with the overall approach of minimizing the mass of the mirror, the mass and volume of the control electronics should also be as low as possible. Rather than attaching a high-voltage amplifier to each channel, a single amplifier is used to control all channels, following the scheme shown in Fig. 11(a). The mirror shape is measured by a wavefront sensor, which then passes the information to a controller.

The mirror controller is based on a design proposed by Song et al. [41]. It consists of a microcontroller whose analog output is amplified to the range -500 to $+500 \mathrm{~V}$ by a single high-voltage amplifier, and then multiplexed into the individual actuator channels that electrically act as capacitors. The controller cycles through each actuator and sets the channel voltage. The voltage level is then held with some minor leakage until the next refresh cycle. This allows control over a large number of actuators by using a single controller board and amplifier board at the expense of control frequency bandwidth, which is ideal for low-frequency disturbances (thermal, creep, etc.). The multiplexing concept is illustrated in Fig. 12, and a photograph of a prototype 42-channel multiplexer is shown in Fig. 11(b).

This controller operates around $1 \mathrm{~Hz}$. The mirror could potentially be operated at higher frequencies, with different electronics; however the implications in terms of heating of the mirror should then be evaluated.

\section{Experiments}

\section{A. Mirror Prototypes}

Although several prototype mirrors were constructed, the results presented in this section were all obtained from the same prototype mirror. This mirror was based on an active laminate consisting of a $200 \mu \mathrm{m}$ thick silicon wafer and a single $20 \mu \mathrm{m}$ thick layer of $\mathrm{P}(\mathrm{VDF}-\mathrm{TrFE})$ coated with segmented annular electrodes, Fig. 13(a). An alternative, finer electrode pattern is also shown in Fig. 13(b). Figure 13(c) shows the whole mirror assembly mounted on nanopositioners that provide piston, tip, and tilt adjustment, and Fig. 13(d) shows the front of the mirror surface with a $1 \overline{00 \mathrm{~nm}}$ reflective gold coating. Note that this mirror prototype was not designed to be thermally balanced, and so even small changes in the laboratory thermal environment can be expected to affect any measurements.

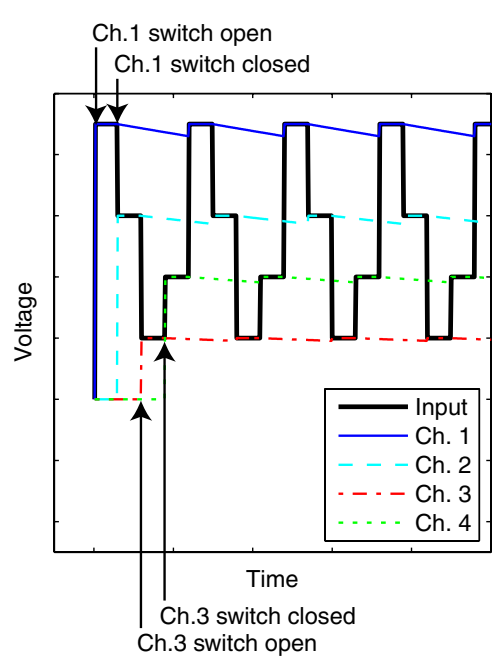

Fig. 12. Traces showing time-varying high-voltage input (heavy black) and multiple actuator channels (light colored) that shows a steady quasi-DC level. Channel decay between refresh points is exaggerated for illustration purposes. 


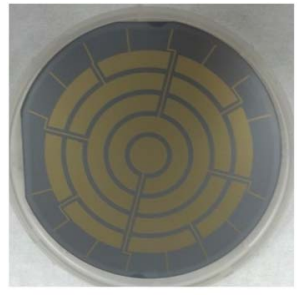

(a)

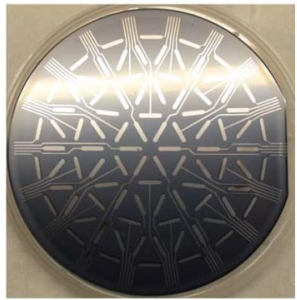

(b)

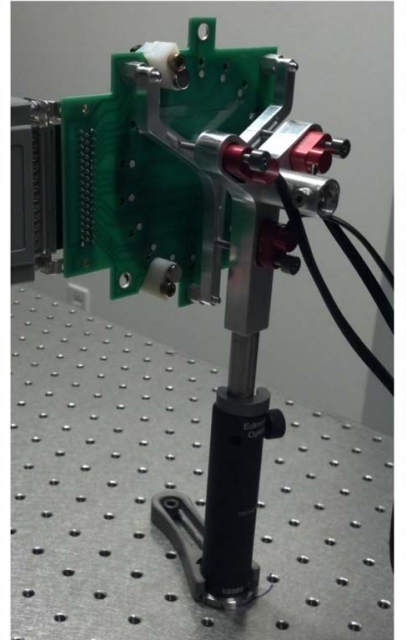

(c)

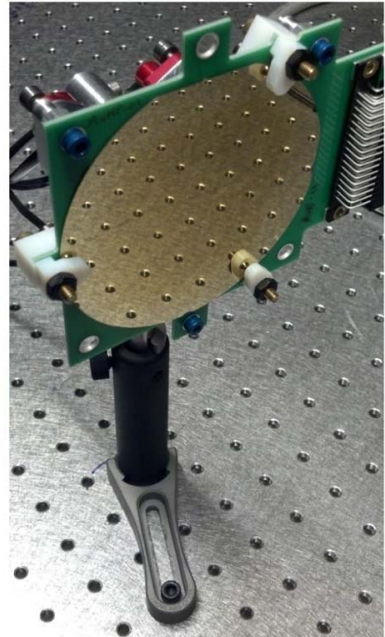

(d)

Fig. 13. Photographs of deformable mirror prototypes: (a) a mirror with a segmented annular electrode pattern, (b) another mirror with a triangular lattice of closely spaced electrodes, (c) rear view of mounted mirror showing three-axis positioning gimbal and mounting board, and (d) front view of mirror showing reflective surface and three low-stress mounting points.

\section{B. Optical Measurement Setup}

In order to measure the shape of the mirror, an optical testbed based around a ThorLabs WFS150-7AR Shack-Hartmann wavefront sensor was used. The sensor utilizes an array of lenslets to form an array of spots on an image sensor; the deviation of the spots from a perfect grid is proportional to the local slope error in the wavefront. Figure 14 shows a diagram of the experimental setup. It consists of a $633 \mathrm{~nm}$ laser beam filtered with a pinhole, collimated, reflected off the deformable mirror under test, and then passed to the wavefront sensor by means of a beam splitter and lens. This arrangement was chosen so as to reimage the mirror pupil to a smaller size that will fit inside the sensor aperture. With a good alignment of all components, the wavefront sensor provides a measurement of the surface figure of the mirror. The Shack-Hartmann sensor had been calibrated by the manufacturer. A thick, flat mirror was used to align the setup; the deformable mirror was substituted in, and the Shack-Hartmann sensor was moved in piston in order to zero the defocus and capture the

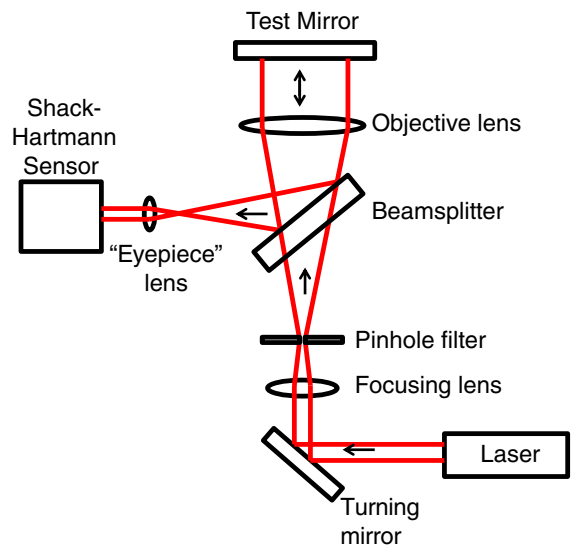

Fig. 14. Schematic diagram of experimental measurement setup. image of the pupil (mirror). In other words, the shape measurement was relative to the closest sphere. The measurement area of the mirror was constrained by the smaller clear aperture of the $75 \mathrm{~mm}$ diameter objective lens.

\section{Influence Functions}

Figure 15(a) shows the measured, individual influence functions from the various channels. The channel numbers are defined in Fig. 16. These measurements were obtained by taking the difference in shape between a reference measurement with all channels off and a new measurement with a single channel turned on and set to $400 \mathrm{~V}$. Figure 15(b) shows the corresponding predictions obtained from the finite-element model described in Section 3.C. Visually, there is a reasonable match between measurements and predictions, but quantitatively the discrepancies are such that experimentally based influence functions are required for accurate shape control. The discrepancies are likely due to beam misalignments as well as thermally induced disturbances of the unbalanced laminate. For example, channel 9 shows evidence of astigmatism in the experimental measurement, due to misalignments.

\section{Focus Control}

This test consisted in using all 16 channels in the mirror to control a single mode. The mode chosen was defocus, i.e., the axisymmetric base curvature component of the mirror surface. A simple, proportional derivative feedback controller was implemented with nonoptimized gains and, for simplicity, the same voltage value was assigned to all channels. An experiment was carried out in which a step defocus change of two waves with a long hold was requested; the step response of the controlled mirror is shown in Fig. 17 , together with the applied voltage of the controller output. 


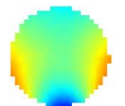

(1)

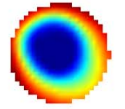

(9)

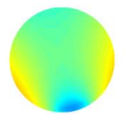

(1)

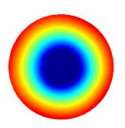

(9)

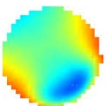

(2)

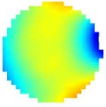

(10)

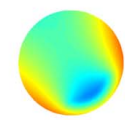

(2)

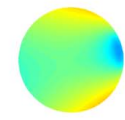

(10)

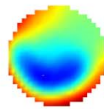

(3)

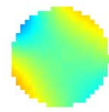

(11)

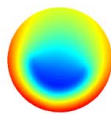

(3)

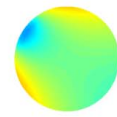

(11)

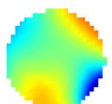

(4)

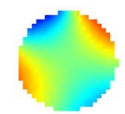

(12)

(a)

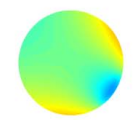

(4)

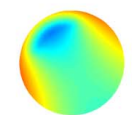

(12)

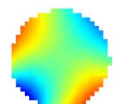

(5)

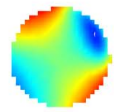

(13)

)

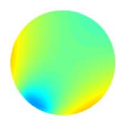

(5)

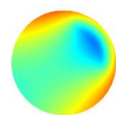

(13)

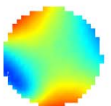

(6)

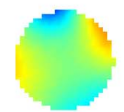

(14)

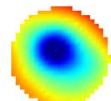

(7)

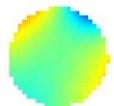

(15)

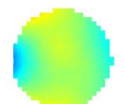

(8)

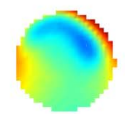

(16)

(b)

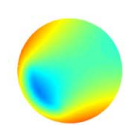

(6)

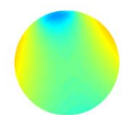

(14)

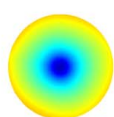

(7)

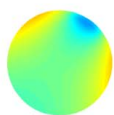

(15)

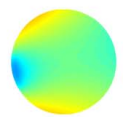

(8)

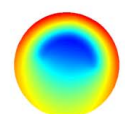

(16)

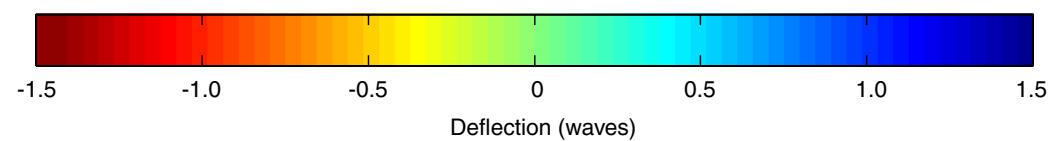

Fig. 15. Influence functions of preliminary $100 \mathrm{~mm}$ mirror prototype with 16 channels with the pattern in Fig. 13 (a). (a) Experimental measurements and (b) finite element predictions due to $400 \mathrm{~V}$ inputs. Deflection color scale is in units of waves at $633 \mathrm{~nm}$. The diameter of the images is only $75 \mathrm{~mm}$ owing to objective lens size constraints.

In the figure, note that after a settling period, dependent on the controller gains, the mirror defocus is controlled well within a small fraction of a wavelength. The long-term drift in the control voltage is in response to the uncontrolled laboratory thermal environment; the oscillatory behavior has not been studied.

\section{E. Multiple Mode Control}

A more complex scenario is to control many response modes by using all of the available actuation channels in order to reduce the mirror aberrations, for example, to make the mirror as flat as possible. In this test, the lowest 66 Zernike modes were minimized in a least-squares sense with 16 independent voltages. The control algorithm was implemented by decomposing each of the 16 measured influence functions

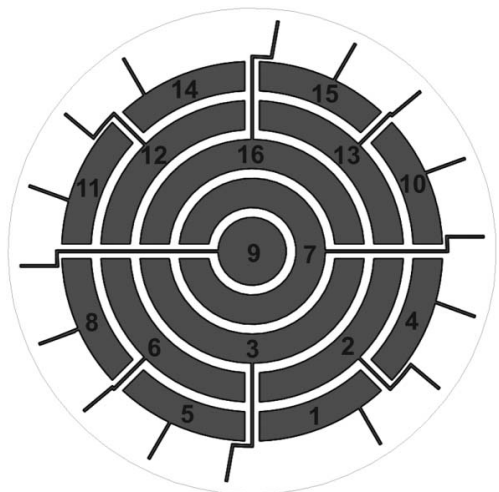

Fig. 16. Definition of actuator numbers in 16-channel prototype. for the mirror into its Zernike components and then implementing a proportional derivative feedback controller that reduces the magnitudes of the measured Zernike components of the actual mirror shape. At each step, the control solution was obtained by computing a constrained (to meet the voltage constraints) least-squares solution of Eq. (6) and multiplying it by a factor less than unity to ensure a damped response without overshoot and to prevent possible material hysteresis effects. The influence functions of the mirror were assumed to be constant and independent of voltage throughout the test.

Figure 18 shows the evolution of the measured RMS error during this test. The initial RMS error was 5.2 waves (at $633 \mathrm{~nm}$ ), which was reduced to

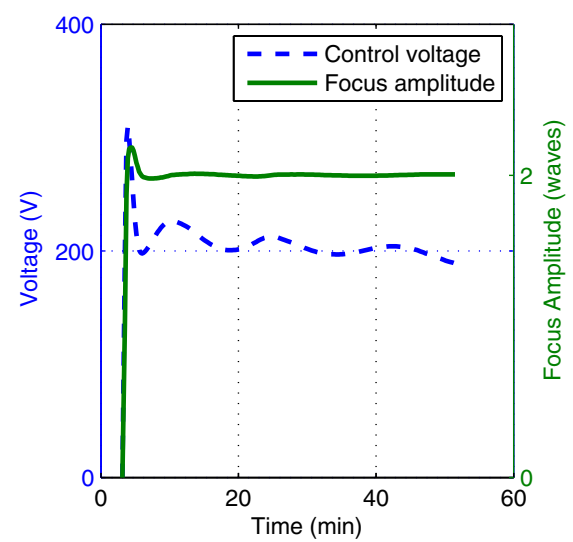

Fig. 17. Demonstration of defocus control using a single input voltage. 


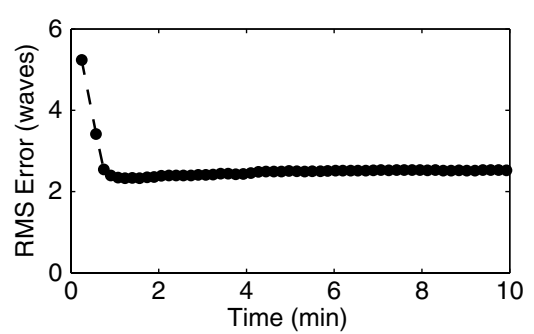

(a)

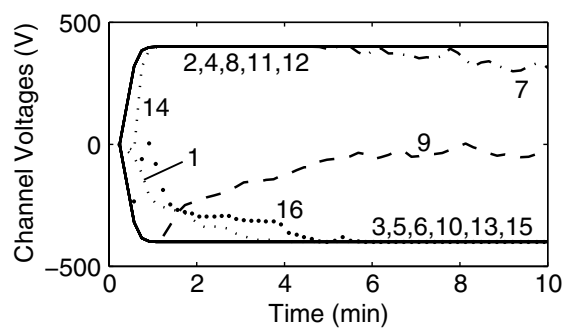

(b)

Fig. 18. (a) Reduction of static RMS shape error using 16 channels and (b) evolution of channel voltages.

about 2.3 waves (an improvement of about $55 \%$ ) in about four steps. The controller was left running for about $10 \mathrm{~min}$ to verify its ability to maintain this low error. Notice that most of the channels hit the controller limits of $\pm 400 \mathrm{~V}$, which indicates that the actuation stroke would first need to be improved in order to further reduce the error. This could be done by switching to a mirror design with a more compliant substrate, by increasing the number of channels in the mirror, by increasing the allowable voltage range, or by using optimized electrode patterns, and potentially by updating the influence functions. The latter approach would require a longer time to achieve convergence because of the delay introduced by remeasuring the influence functions. The controller response time could be decreased to compensate for this slow down.

\section{Discussion and Conclusion}

The concept, design, and low-cost fabrication methods of ultralightweight, deformable mirrors have been presented in this paper. After an initial investigation of candidate substrate and piezoelectric materials, silicon and PVDF copolymer were selected for further study, and a design formula for the maximum curvature change as a function of the blocked stress of the piezoelectric and the thickness and modulus of the substrate was obtained. A minimum thickness bound on the substrate was established by considering buckling of the laminate mirror during poling, and a general design chart for $20 \mu \mathrm{m}$ thick PVDF copolymer active layers on different substrates and thicknesses was derived. Two different actuation patterns were studied with a finite-element model, and three different densities of the actuators were considered. Correctability and stroke of the mirror were evaluated for each design, providing the basis for future optimization.
From these studies, simple prototype mirrors were designed and built using silicon substrates with a diameter of $100 \mathrm{~mm}$ and areal density of $0.6 \mathrm{~kg} / \mathrm{m}^{2}$ and were measured to be capable of $20 \mu \mathrm{m}$ of displacement with current geometries and materials. The present solutions may be scaled to larger sizes by using larger silicon wafers or different substrates such as glass. A kinematic mounting scheme for these thin, flexible mirrors has been introduced and demonstrated in the laboratory. This scheme may be suitable for a variety of operational environments, but launch survivability or vibrations during operation may require refinements such as a releasable restraint system and/or shunted piezoelectric damping provided by the actuators.

Initial optical testing has been performed on a mirror with 16 active channels, and the actuator influence functions have been measured and found to match reasonably well to the model predictions. On this preliminary prototype, the residual wavefront error was reduced by about $50 \%$ to two waves (RMS wavefront error at $633 \mathrm{~nm}$ ). Hence it is expected that diffraction-limited performance can be achieved with design optimization and refinement of the proposed concept.

We thank Dr. Harish Manohara (JPL) for providing access to the Microdevices Lab (MDL) cleanroom facilities for sample fabrication. We thank Dr. Risaku Toda (JPL) and Mr. Victor White (JPL) for processing equipment training and usage advice at the MDL. We also thank Dr. Namiko Yamamoto (Caltech) for assistance and advice on mirror fabrication. We are grateful to Dr. Jim Breckinridge (Caltech) for helpful discussions on optics and telescope design, Prof. Chiara Daraio (Caltech) and John Steeves (Caltech) for advice regarding the fabrication of prototype mirrors, and Dr. Andrew Shapiro (JPL) for advice on material selection. We also appreciate the fabrication facilities provided by the Kavli Nanoscience Institute at Caltech. Financial support from the Keck Institute of Space Studies (KISS) at Caltech is gratefully acknowledged. A part of this research was carried out at the Jet Propulsion Laboratory, California Institute of Technology, under a contract with the National Aeronautics and Space Administration (NASA).

\section{References}

1. H. P. Stahl, "Design study of 8 meter monolithic mirror UV/optical space telescope," Proc. SPIE 7010, 701022 (2008).

2. J. P. Gardner, J. C. Mather, M. Clampin, R. Doyon, M. A. Greenhouse, H. B. Hammel, J. B. Hutchings, P. Jakobsen, S. J. Lilly, K. S. Long, J. I. Lunine, M. J. McCaughrean, M. Mountain, J. Nella, G. H. Rieke, M. J. Rieke, H.-W. Rix, E. P. Smnith, G. Sonneborn, M. Staivelli, H. S. Stockman, R. A. Windhorst, and G. S. Wright, "The James Webb space telescope," Space Sci. Rev. 123 485-606 (2006).

3. C. M. Mountain, "The future of ELTS (Extremely Large Telescopes), a personal view," Proc. SPIE 5382, 763-770 (2004).

4. J. B. Breckinridge, J. Dooley, M. Ortiz, and S. Pellegrino, "Large space apertures (LSA) study report" (Keck Institute of Space Studies, 2009).

5. J. G. Katz, "Estimation and control of flexible space structures for autonomous on-orbit assembly," M.S. thesis (MIT, 2009). 
6. M. C. Natori and K. Ukegawa, "Concept of self-assembly of space structure systems using autonomous modules," in 54th International Astronautical Congress of the International Astronautical Federation (American Institute of Aeronautics and Astronautics, 2003), paper IAC-03-U.1.01.

7. L. P. Rodgers, "Concepts and technology development for the autonomous assembly and reconfiguration of modular space systems," M.S. thesis (MIT, 2005).

8. C. Underwood and S. Pellegrino, "Autonomous assembly of a reconfigurable space telescope (AAReST) for astronomy and Earth observation," presented at 8th IAA Symposium on Small Satellites for Earth Observation, Berlin, 4-8 April 2011.

9. G. Hickey, T. Barbee, M. Ealey, and D. Redding, "Actuated hybrid mirrors for space telescopes," Proc. SPIE 7731, 773120 (2010).

10. K. Patterson and S. Pellegrino, "Shape correction of thin mirrors," presented at 52nd AIAA/ASME/ASCE/AHS/ASC Structures, Structural Dynamics and Materials Conference, Denver, Colorado, 4-7 April 2011.

11. K. Patterson, N. Yamamoto, and S. Pellegrino, "Thin deformable mirrors for a reconfigurable space aperture" in 53rd AIAA/ASME/ASCE/AHS/ASC Structures, Structural Dynamics and Materials Conference (American Institute of Aeronautics and Astronautics, 2012), paper AIAA-2012-1668.

12. A. Norton, J. W. Evans, D. Gavel, D. Dillon, D. Palmer, B. Macintosh, K. Morzinski, and S. Cornelissen, "Preliminary characterization of Boston Micromachines' 4096-actuator deformable mirror," Proc. SPIE 7209, 72090I (2009).

13. A. Tokovinin, S. Thomas, and G. Vdovin, "Using $50 \mathrm{~mm}$ electrostatic membrane deformable mirror in astronomical adaptive optics," Proc. SPIE 5490, 580-585 (2004).

14. E. H. Yang, Y. Hishinuma, J.-G. Cheng, S. Trolier-McKinstry, E. Bloemhof, and B. M. Levine, "Thin-film piezoelectric unimorph actuator-based deformable mirror with a transferred silicon membrane," J. Microelectromech. Syst. 15, 1214-1225 (2006).

15. T. Bruno, "Deformable mirrors," Northrop Grumman, accessed 11 Jan. 2013, http://132.228.182.183/businessventures/ aoa-xin/deformable_mirrors/index.html.

16. P. Salinari, C. Del Vecchio, and V. Biliotti, "A study of an adaptive secondary mirror," in Proceedings of the ICO-16 (International Commission for Optics) Satellite Conference on Active and Adaptive Optics, Vol. 48 of European Southern Observatory Conference and Workshop Proceedings (European Southern Observatory, 1994), p. 247.

17. J. Lindler and E. Flint, "Robustness of thin film shells with discrete boundary actuation," in 47th AIAA/ASME/ASCE/ AHS/ASC Structures, Structural Dynamics, and Materials Conference (American Institute of Aeronautics and Astronautics, 2006), paper AIAA 2006-1904.

18. M. Laslandes, E. Hugot, M. Ferrari, C. Hourtoule, C. Singer, C. Devilliers, C. Lopez, and F. Chazallet, "Mirror actively deformed and regulated for applications in space: design and performance," Opt. Eng. 52, 091803 (2013).

19. E. Steinhaus and S. Lipson, "Bimorph piezoelectric flexible mirror," J. Opt. Soc. Am. 69, 478-481 (1979).

20. T. Sato, H. Ishida, and O. Ikeda, "Adaptive PVDF piezoelectric deformable mirror system," Appl. Opt. 19, 1430-1434 (1980).

21. C. P. Kuo, "A deformable mirror concept for adaptive optics in space," Proc. SPIE 1542, 420-433 (1991).

22. Q. Chen, D. Natale, B. Neese, K. Ren, M. Lin, Q. M. Zhang, M. Pattom, K. W. Wang, H. Fang, and E. Im, "Piezoelectric polymers actuators for precise shape control of large scale space antennas," Proc. SPIE 6524, 65241P (2007).
23. D. D. Pearson, J. L. Cavaco, and J. Roche, "Multichannel, surface parallel, zonal transducer system," U.S. patent 7,683,524 B2 (March 23, 2010).

24. J. Pearson, J. Moore, and H. Fang, "Large and high precision inflatable membrane reflector," in 51st AIAA/ASME/ASCE / AHS/ASC Structures, Structural Dynamics, and Materials Conference (American Institute of Aeronautics and Astronautics, 2010), paper AIAA-2010-2500.

25. R. Bastaits, G. Rodrigues, P. Jetteur, P. Hagedorn, and A. Preumont, "Multi-layer adaptive thin shells for future space telescopes," Smart Mater. Struct. 21, 064004 (2012).

26. B. de Blonk, J. Moore, B. Patrick, and E. Flint, "Membrane mirrors in space telescopes," in Recent Advances in Gossamer Spacecraft, C. Jenkins, ed. (American Institute of Aeronautics and Astronautics, 2006), pp. 45-108.

27. J. E. Huber, N. A. Fleck, and M. F. Ashby, "The selection of mechanical actuators based on performance indices," Proc. R. Soc. London 453, 2185-2205 (1997).

28. R. A. Kellogg and A. B. Flatau, "Blocked force investigation of a Terfenol-D transducer," Proc. SPIE 3668, 184-195 (1999).

29. W. K. Wilkie, R. G. Bryant, J. W. High, R. L. Fox, R. F Hellbaum, A. Jalink Jr., B. D. Little, and P. H. Mirick, "Low-cost piezocomposite actuator for structural control applications," Proc. SPIE 3991, 323-334 (2000).

30. V. Cotroneo, W. N. Davis, V. Marquez, P. B. Reid, D. A Schwartz, R. L. Johnson-Wilke, S. E. Trolier-McKinstry, and R. H. T. Wilke, "Adjustable grazing incidence $x$-ray optics based on thin PZT films," Proc. SPIE 8503, 850309 (2012).

31. T. R. Dargaville, M. C. Celina, J. M. Elliott, P. M. Chaplya, G. D. Jones, D. M. Mowery, R. A. Assink, R. L. Clough, and J. W. Martin, "Characterization, performance and optimization of PVDF as a piezoelectric film for advanced space mirror concepts," Sandia National Laboratories Report SAND20056846 (Sandia National Laboratories, 2005).

32. M. Horányi, V. Hoxie, D. James, A. Poppe, C. Bryant, B. Grogan, B. Lamprecht, J. Mack, F. Bagenal, S. Batiste, N. Bunch, T. Chanthawanich, F. Christensen, M. Colgan, T. Dunn, G. Drake, A. Fernandez, T. Finley, G. Holland, A. Jenkins, C. Krauss, E. Krauss, O. Krauss, M. Lankton, C. Mitchell, M. Neeland, T. Reese, K. Rash, G. Tate, C. Vaudrin, and J. Westfall, "The Student Dust Counter on the New Horizons Mission," Space Sci. Rev. 140, 387-402 (2008).

33. G. G. Stoney, "The tension of metallic films deposited by electrolysis," Proc. R. Soc. London Ser. A 82, 172-175 (1909).

34. L. B. Freund, "Substrate curvature due to thin film mismatch strain in the nonlinear deformation range," J. Mech. Phys. Solids 48, 1159-1174 (2000).

35. L. B. Freund and S. Suresh, Thin Film Materials, Stress, Defect Deformation and Surface Evolution (Cambridge University, 2003).

36. M. Born and E. Wolf, Principles of Optics (Pergamon, 1989).

37. W. W. Zhang, "Manufacture of mirror glass substrates for the NUSTAR mission," Proc. SPIE. 7437, 74370N (2009).

38. Y. Ezoe, T. Shirata, I. Mitsuishi, M. Ishida, K. Mitsuda, K. Morishita, and K. Nakajima, "Shaped silicon wafers obtained by hot plastic deformation: performance evaluation for future astronomical x-ray telescopes," Appl. Opt. 48, 3830-3838 (2009).

39. P. Bely, The Design and Construction of Large Optical Telescopes (Springer, 2003).

40. R. Klein, Concrete and Abstract Voronoi Diagrams (Springer-Verlag, 1987).

41. H. Song, A. Simonov, and G. Vdovin, "Multiplexing control of a multichannel piezoelectric deformable mirror," Proc. SPIE 6018, 60181F (2005). 